Regards sur l'économie allemande

Bulletin économique du CIRAC

$101 \mid 2011$

Varia

\title{
Compétitivité : Flexibilité et stabilité des entreprises
}

Solène Hazouard

\section{OpenEdition}

1 Journals

Édition électronique

URL : http://journals.openedition.org/rea/4299

DOI : 10.4000/rea.4299

ISBN : 978-2-8218-0954-3

ISSN : 1965-0787

\section{Éditeur}

CIRAC

Édition imprimée

Date de publication : 10 juin 2011

Pagination : 38

ISSN : 1156-8992

\section{Référence électronique}

Solène Hazouard, "Compétitivité : Flexibilité et stabilité des entreprises ", Regards sur l'économie allemande [En ligne], 101 | juin 2011, mis en ligne le 19 septembre 2011, consulté le 15 septembre 2020. URL : http://journals.openedition.org/rea/4299 
Flexibilité et stabilité sont complémentaires

5 profils d'entreprises identifiés...

... dont les spécificités influent sur l'équilibre flexibilité/stabilité

\section{$\ni$ Compétitivité : Flexibilité et stabilité des entreprises}

Durant la crise, les entreprises industrielles allemandes ont fait preuve d'un haut degré de flexibilité (voir dans ce numéro l'analyse d'l. Zapf) qui leur a permis de préserver, voire de développer leur compétitivité hors prix. Cette flexibilité comprend plusieurs dimensions. Outre l'aptitude (vitale en temps de crise) à adapter les volumes et les capacités de production à une demande fortement instable, le succès des entreprises sur le long terme dépend de trois facteurs complémentaires : la capacité à effectuer des adaptations produit sur mesure en fonction de la demande, celle de développer et de fabriquer rapidement des produits innovants conformes aux souhaits du client, et la faculté de proposer des délais de livraison plus courts et plus fiables que la concurrence. Cette flexibilité doit cependant s'accompagner d'une certaine stabilité afin de motiver durablement les salariés et de stimuler leur créativité - par le partage de savoir-faire, de normes, ou encore par une culture d'entreprise. Dès lors, flexibilité et stabilité agissent comme des forces complémentaires, précisent $\mathrm{S}$. Kinkel et $\mathrm{S}$. Maloca dans une analyse parue en décembre 2010 et qui présente les résultats de l'enquête Modernisation de la production 2009 de l'Institut Fraunhofer de Recherche sur les Systèmes et l'Innovation (ISI).

Menée, dans le cadre du projet VITNESS du ministère fédéral de la Formation et de la Recherche (BMBF), auprès de quelque 1500 entreprises industrielles tous secteurs confondus, l'enquête du Fraunhofer ISI a permis d'identifier 5 profils d'entreprises en fonction de leur orientation stratégique. Les deux premiers groupes sont orientés vers la flexibilité, qu'il s'agisse de l'adaptation produit ( $27 \%$ des entreprises) ou de l'innovation produit $(11 \%)$. Les groupes 3 et $4:$ : Rentabilité et tenue des délais de livraison » $(16 \%$ des entreprises) et "Stabilité des process et qualité " $(25 \%)$ placent nettement le curseur sur la stabilité, tandis que le cinquième profil : "Différenciation sur des marchés de niche » (21\% des entreprises), n'est orienté ni vers la flexibilité, ni vers la stabilité.

Les divergences entre ces 5 profils portent également sur les facteurs de flexibilité interne et externe des entreprises sondées, leur performance économique et leur comportement innovant. Ainsi le profil « Flexibilité par l'adaptation produit » privilégie les éléments de flexibilité interne (comptes épargne temps, recours à une main-d'œuvre qualifiée, minimisation des coûts fixes de production). Si ces entreprises se prêtent à des concessions quant à l'efficience et à la qualité de leurs process, elles réalisent de larges profits, ainsi que de bonnes performances en termes d'innovation. Le profil « Flexibilité par l'innovation produit » se caractérise par une maîtrise de la chaîne de création de valeur, des moyens élevés consacrés à la R\&D, de nombreuses innovations et, partant, un bon rendement. Le profil "Rentabilité et délais de livraison » mise prioritairement sur des process efficients et sur les éléments de flexibilité externe (emploi intérimaire, soustraitance). Malgré un haut degré de productivité, ces entreprises réalisent peu de bénéfices en raison d'une pression élevée sur les coûts. Le profil "Stabilité des process et qualité » utilise peu les outils de flexibilité interne et externe. II s'assure un niveau de profits stable via une performance stable en termes de qualité. Enfin, avec une productivité moyenne et un recours également moyen aux éléments de flexibilité interne et externe, les entreprises du profil " Différenciation sur des marchés de niche ", atteignent un bon rendement au sein de niches "confortables". A la lumière de ces 5 profils distincts apparaît la nécessité de moduler le recours aux leviers internes et externes de flexibilité et de stabilité, afin d'obtenir l'équilibre souhaité pour l'entreprise. $(\mathrm{SH})$

Performance des profils d'entreprises identifiés

\begin{tabular}{|c|c|c|c|c|c|c|c|c|}
\hline \multirow[b]{2}{*}{ Profil } & \multicolumn{2}{|c|}{ Flexibilité } & \multicolumn{2}{|c|}{ Stabilité } & \multicolumn{2}{|c|}{ Rentabilité } & \multicolumn{2}{|c|}{ Innovation } \\
\hline & Interne & Externe & $\begin{array}{l}\text { Respect } \\
\text { des délais }\end{array}$ & Qualité & Productivité & Rendement & Input & Output \\
\hline $\begin{array}{l}1 \text { : Flexibilité par l'adaptation } \\
\text { produit }\end{array}$ & $\uparrow$ & $\rightarrow$ & $\downarrow$ & y & $y$ & $\rightarrow$ & $\rightarrow$ & $\pi$ \\
\hline $\begin{array}{l}2 \text { : Flexibilité par l'innovation } \\
\text { produit }\end{array}$ & $\pi$ & ע & $\rightarrow$ & $\downarrow$ & $\uparrow$ & $\uparrow$ & $\uparrow$ & $\uparrow$ \\
\hline $\begin{array}{l}3: \text { Rentabilité et délais de } \\
\text { livraison }\end{array}$ & $\downarrow$ & $\uparrow$ & $\uparrow$ & $\uparrow$ & $\uparrow$ & $\downarrow$ & $\downarrow$ & $\downarrow$ \\
\hline $\begin{array}{l}4: \text { Stabilité des process et } \\
\text { qualité }\end{array}$ & $\downarrow$ & $\downarrow$ & $\rightarrow$ & 个 & $\rightarrow$ & $\rightarrow$ & $y$ & $\rightarrow$ \\
\hline $\begin{array}{l}5 \text { : Différenciation sur des } \\
\text { marchés de niche }\end{array}$ & $\rightarrow$ & y & $\rightarrow$ & $\uparrow$ & $\downarrow$ & $\uparrow$ & $\rightarrow$ & $\rightarrow$ \\
\hline
\end{tabular}

Source: Enquête Modernisation de la production 2009, Institut Fraunhofer ISI, Mitteilungen aus der ISI-Erhebung, $\mathrm{n}^{\circ} 54$, décembre 2009. 